\title{
Crystallographic insights into the structural aspects of thioctic acid based halogen-bond donor for the functionalization of gold nanoparticles
}

\section{Kavitha Buntara Sanjeeva, Ilaria Tirotta, Vijith Kumar, Francesca Baldelli Bombelli, Giancarlo Terraneo and Pierangelo Metrangolo}

Acta Cryst. (2017). B73, 240-246

\section{IIUCr Journals CRYSTALLOGRAPHY JOURNALS ONLINE \\ Copyright (C) International Union of Crystallography \\ Author(s) of this paper may load this reprint on their own web site or institutional repository provided that this cover page is retained. Republication of this article or its storage in electronic databases other than as specified above is not permitted without prior permission in writing from the IUCr. \\ For further information see http://journals.iucr.org/services/authorrights.html}




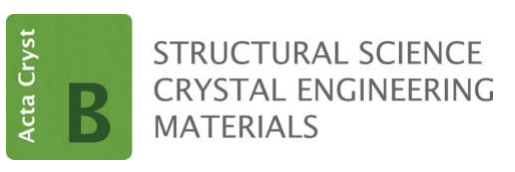

ISSN 2052-5206

Received 30 January 2017

Accepted 24 February 2017

Edited by M. Erdelyi, University of Gothenburg, Sweden

Keywords: self-assembly; halogen bonding; gold nanoparticles; surface functionalization.

CCDC references: 1529898; 1529897

Supporting information: this article has supporting information at journals.iucr.org/b

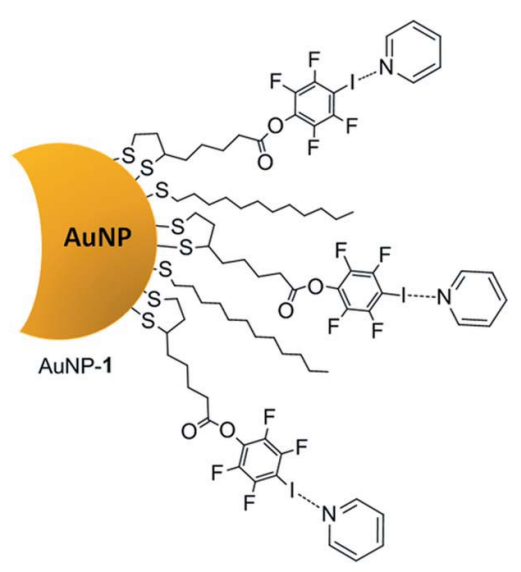

C 2017 International Union of Crystallography

\section{Crystallographic insights into the structural aspects of thioctic acid based halogen-bond donor for the functionalization of gold nanoparticles}

\author{
Kavitha Buntara Sanjeeva, ${ }^{a}$ Ilaria Tirotta, ${ }^{a}$ Vijith Kumar, ${ }^{a}$ Francesca Baldelli \\ Bombelli, $^{a *}$ Giancarlo Terraneo $^{\mathrm{a} *}$ and Pierangelo Metrangolo ${ }^{\mathrm{a}, \mathrm{b}}$

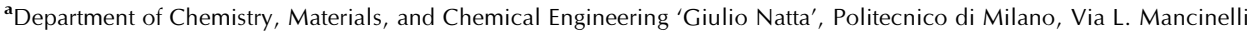 \\ 7, 20131 Milano, Italy, and ${ }^{\mathbf{b}}$ HYBER Centre of Excellence, Department of Applied Physics, Aalto University, PO Box \\ 15100, FI-02150 Espoo, Finland. *Correspondence e-mail: francesca.baldelli@polimi.it, giancarlo.terraneo@polimi.it
}

The synthesis and self-assembly capabilities of a new halogen-bond donor ligand, 2,3,5,6-tetrafluoro-4-iodophenyl 5-(1,2-dithiolan-3-yl)pentanoate (1), are reported. The crystal structure of ligand (1) and the formation of a cocrystal with 1,2-di(4-pyridyl)ethylene, (1).(2), both show halogen bonds involving the 4iodotetrafluorobenzene moiety. Ligand (1), being a self-complementary unit, forms an infinite halogen-bonded chain driven by the S...I synthon, while the cocrystal (1).(2) self-assembles into a discrete trimeric entity driven by the N $\cdots I$ synthon. Ligand (1) was also successfully used to functionalize the surface of gold nanoparticles, AuNP-(1). Experiments on the dispersibility profile of AuNP-(1) demonstrated the potential of halogen bonding in facilitating the dispersion of modified NPs with halogen-bond donors in pyridine.

\section{Introduction}

Metallic nanoparticles (NPs) attracted considerable interest in different scientific communities due to their unique optical, electronic, magnetic and catalytic properties (Burda et al., 2005; Pankhurst et al., 2003; Astruc et al., 2005). Several classes of engineered NPs with different metallic cores have been developed and used (i.e. $\mathrm{Ag}, \mathrm{Au}, \mathrm{Co}, \mathrm{Cu}, \mathrm{Fe}$ etc.; Rycenga et al., 2011; Gupta \& Gupta, 2005; Khanna et al., 2007; Wu et al., 2015), however, gold NPs (AuNPs) gained a dominant role among these nanosystems and have been extensively exploited as multifunctional platforms for applications in physics, chemistry, biology, medicine and material science (Daniel \& Astruc, 2004; Saha et al., 2012; Ghosh et al., 2008). In fact, in the last two decades a tremendous synthetic effort has led to the production of various gold chemical nanoobjects with different functionalized surfaces (Grzelczak et al., 2008; Sperling \& Parak, 2010). In general, the chemical nature of gold functionalized nanosurfaces strongly affect their physical-chemical properties and self-assembly behaviour together with the size and shape of the gold cores (Xu et al., 2013). The surface ligand shell is the anchoring point for attaching other components to the NPs - be they molecules, surfaces or even other NPs - and thus controls and regulates the interfacial properties and organization of AuNPs.

Stabilization of AuNPs in solution and their organization in superlattices are fundamental challenges in this field and even if the full control of these properties is far from being achieved, several advances have been made using different supramolecular approaches. Molecular self-assembly is driven by non-covalent interactions such as ionic, hydrophobic, van der Waals and hydrogen bonds (HBs), and these same inter- 
actions are involved in NP self-assembly, which is predicted by the Derjaguin, Landau, Veruley \& Overbeak (DLVO) theory (Derjaguin \& Sidorenkov, 1941; Verwe \& Overbeek, 1948), considering its limitations when it is applied to nano-scaled objects (Li et al., 2013; Yang et al., 2014; Coulston et al., 2011; Zhang et al., 2016). Hence, by clever design of the surface chemistry and structural properties of AuNPs it is possible to exploit supramolecular strategies for controlling the equilibrium between aggregated and non-aggregated states of AuNPs. Among all the non-covalent interactions the hydrogen bond is the most used supramolecular tool for tuning AuNP self-assembly in solution, in fact, gold surfaces have been functionalized with several $\mathrm{HB}$ donors and acceptors that span from small molecules to polymers (Vuluga et al., 2008; Hao \& Lian, 2000; Heo et al., 2013).

As a counterpart of the hydrogen bond, the halogen bond (XB) has consolidated its role as a fundamental tool in constructing well organized supramolecular architectures and thanks to its strength, directionality and tunability has been applied in different research fields such as anion sensing, soft and functional materials, catalysis, solid-state reactivity and biological systems (Cavallo et al., 2016). Although the ability of $\mathrm{XB}$ in controlling self-assembly phenomena is well recognized, its employment as a driving force in the organization of AuNPs is confined to a few examples. Specifically, van der Boom and co-workers have reported the functionalization of AuNPs with XB-donating stilbene derivatives, which formed either chain-like structures or large, dense assemblies, upon mixing with bidentate $\mathrm{XB}$ acceptors depending on the concentration of the bipyridyl cross-linker (Shirman et al., 2010). XB was also used, by the same group, to prepare surface-confined nanostructures attaching the XB donor NPs onto silicon and glass substrates functionalized with pyridine groups (XB acceptors; Shirman et al., 2011).

On the basis of these results in which the ability of $\mathrm{XB}$ to regulate the aggregation of functionalized gold nanosystems was highlighted, we prepared a new XB donor ligand (1) based on thioctic acid for surface functionalization of AuNPs. Specifically, we first report here the synthesis of the XB donor (1) and the study, in the solid state, of its assembling capabilities with the XB acceptor [1,2-di(4-pyridyl)ethylene, (2)]. Ligand (1) was then successfully used to functionalize gold nanosurfaces for producing halogen-bonding donor NPs,

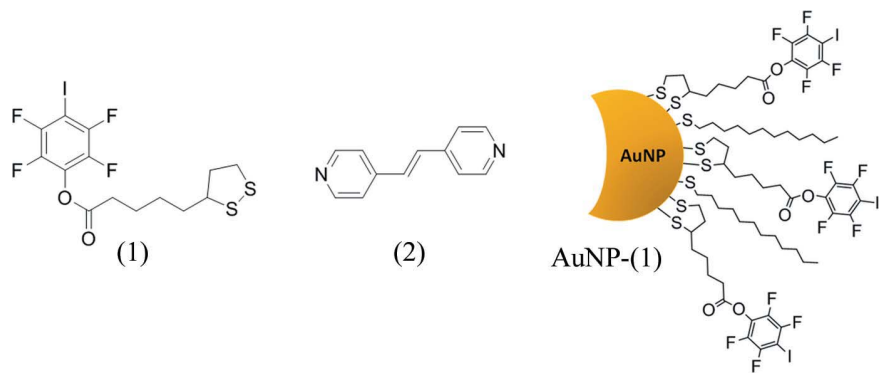

Figure 1

Chemical structure of the XB donor ligand (1) and XB acceptor (2) and schematic representation of AuNP-(1).
AuNP-(1) (Fig. 1). In addition, a preliminary study on the dispersibility of AuNP-(1) in different solvents was performed to show the different behaviour of AuNP-(1) compared with fully alkylated AuNPs (AuNP-DT), highlighting the interaction of the $\mathrm{XB}$ donors on the gold surface with solvent molecules with $\mathrm{XB}$ acceptor properties.

\section{Experimental}

\subsection{Materials and methods}

All chemicals were of reagent-grade quality obtained from commercial sources and used without further purification. Anhydrous solvents were used for the synthesis of XB donor ligand (1). Melting points were determined with an Olympus BX51 polarized optical microscope equipped with a Linkam Scientific LTS-350 heating stage. ${ }^{1} \mathrm{H}$ NMR, ${ }^{13} \mathrm{C}$ NMR and ${ }^{19} \mathrm{~F}$ NMR spectra were recorded at ambient temperature with a Bruker 400 and $500 \mathrm{MHz}$ spectrometer. ${ }^{1} \mathrm{H}$ and ${ }^{13} \mathrm{C}$ chemical shifts were measured relative to internal TMS and all chemical shift $(\delta)$ values are given in p.p.m. and coupling constants $(J)$ are in Hz. FT-IR, UV-vis, transmission electron microscopy (TEM), dynamic light scattering (DLS) and inductively coupled plasma atomic emission spectroscopy (ICP-AES) analysis are reported in the supporting information.

\subsection{Synthesis of ligand 2,3,5,6-tetrafluoro-4-iodophenyl 5- (1,2-dithiolan-3-yl)pentanoate (1)}

Iodopentafluorobenzene $(34 \mathrm{mmol})$ and $\mathrm{KOH}(102 \mathrm{mmol})$ in $t$ - $\mathrm{BuOH}(311.4 \mathrm{mmol})$ were refluxed in an oil bath at $363 \mathrm{~K}$ for $6.5 \mathrm{~h}$. Hydrochloric acid $(5 \%, 20 \mathrm{ml})$ was added and aqueous $t$ - $\mathrm{BuOH}$ was distilled off under vacuum. The residue was acidified to $\simeq \mathrm{pH} 2$ using $5 \%$ aqueous $\mathrm{HCl}$. The filtrate was extracted with diethyl ether and dried over anhydrous sodium sulfate. The solvent was removed under reduced pressure, and the 2,3,5,6-tetrafluoro-4-iodophenol was obtained as a white solid. Yield $=5.2 \mathrm{~g}(50 \%) .{ }^{1} \mathrm{H}$ NMR $\left(400 \mathrm{MHz}, \mathrm{CDCl}_{3}\right) \delta=5.96$ (s, 1H) p.p.m. ${ }^{19} \mathrm{~F} \mathrm{NMR}(500 \mathrm{MHz}$, $\left.\mathrm{CDCl}_{3}\right) \delta=-122.5(2 \mathrm{~F}),-161.1(2 \mathrm{~F})$ p.p.m. MS: molecular ion peak $m / z 290.7$.

2,3,5,6-Tetrafluoro-4-iodophenol (1.71 mmol), thioctic acid (1.71 mmol), 1,3-dicyclohexylcarbodiimide $(1.88 \mathrm{mmol})$ and 4 $(N, N$-dimethylamino $)$ pyridine $(0.044 \mathrm{mmol})$ were dissolved in dry dichloromethane $(15 \mathrm{ml})$ and stirred at room temperature for $48 \mathrm{~h}$. The precipitate was filtered off and the filtrate was washed with $5 \%(30 \mathrm{ml})$ aqueous acetic acid, and the organic layer was separated and concentrated under reduced pressure. The crude sample was purified by column chromatography using hexane/EtOAc (8:2) as an eluent. The XB donor ligand (1) was obtained as a yellow solid. Yield $=0.51 \mathrm{~g}(62 \%) .{ }^{1} \mathrm{H}$ NMR $\left(\mathrm{CDCl}_{3}, 400 \mathrm{MHz}\right) \delta=3.59(\mathrm{~m}, 1 \mathrm{H}), 3.14(\mathrm{~m}, 2 \mathrm{H}), 2.68$ $(\mathrm{t}, 2 \mathrm{H}), 2.47(\mathrm{~m}, 1 \mathrm{H}), 1.95(\mathrm{~m}, 1 \mathrm{H}), 1.83(\mathrm{~m}, 2 \mathrm{H}), 1.74(\mathrm{~m}, 2 \mathrm{H})$, $1.60(\mathrm{~m}, 2 \mathrm{H})$ p.p.m. ${ }^{19} \mathrm{~F} \mathrm{NMR}\left(\mathrm{CDCl}_{3}, 500 \mathrm{MHz}\right) \delta=-120.9$ (2F), -151.8 (2F) p.p.m. ${ }^{13} \mathrm{C} \mathrm{NMR}\left(\mathrm{CDCl}_{3}, 400 \mathrm{MHz}\right): \delta=$ 168.9, 148.4, 145.8, 141.4, 139, 129.6, 68.8, 56.1, 40.1, 38.5, 34.4, 33.1, 28.4, 24.5 p.p.m. FT-IR ( $\mathrm{cm}^{-1}$, selected bands): 2929.8, 1771, 1479.7, 1112.4, 966.7. $\mathrm{C}_{14} \mathrm{H}_{13} \mathrm{~F}_{4} \mathrm{I}_{1} \mathrm{O}_{2} \mathrm{~S}_{2}[M]^{+}$480.3, 
Table 1

Experimental details for ligand (1) and cocrystal (1).(2).

For all structures: triclinic, $P \overline{1}$. Experiments were carried out at $103 \mathrm{~K}$ with Mo $K \alpha$ radiation using a Bruker APEXII CCD. Absorption was corrected for by multi-scan methods ( $S A D A B S)$. H-atom parameters were not refined.

(1)

Crystal data

CCDC number

Chemical formula

$M_{\mathrm{r}}$

$a, b, c(\AA)$

$\alpha, \beta, \gamma\left({ }^{\circ}\right)$

$V\left(\AA^{3}\right)$

$Z$

$\mu\left(\mathrm{mm}^{-1}\right)$

Crystal size $\left(\mathrm{mm}^{3}\right)$

Data collection

$T_{\min }, T_{\max }$

No. of measured, independent and observed $[I>2 \sigma(I)]$ reflections

$R_{\text {int }}$

$(\sin \theta / \lambda)_{\max }\left(\AA^{-1}\right)$

1529898

$\mathrm{C}_{14} \mathrm{H}_{13} \mathrm{~F}_{4} \mathrm{I}_{1} \mathrm{O}_{2} \mathrm{~S}_{2}$

480.26

5.1915 (7), $11.1729(14), 15.037(2)$

105.837 (6), 90.773 (6), 100.810 (5)

822.30 (19)

2

2.25

$0.18 \times 0.12 \times 0.04$

$0.636,0.746$

$24056,4154,3591$

0.031

0.684

$0.034,0.077,1.05$

4154

221

15

$-0.65,1.50$

$\Delta \rho_{\min }, \Delta \rho_{\max }\left(\mathrm{e} \AA^{-3}\right)$

Computer programs: APEX2, SAINT (Bruker, 2012), SHELXS (Sheldrick, 2008), ORTEP3 (Farrugia, 1997), Mercury (Macrae et al., 2006).
$(1) \cdot(2)$

$$
\begin{aligned}
& 1529897 \\
& 2 \mathrm{C}_{14} \mathrm{H}_{13} \mathrm{~F}_{4} \mathrm{IO}_{2} \mathrm{~S}_{2} \cdot \mathrm{C}_{12} \mathrm{H}_{10} \mathrm{~N}_{2} \\
& 1142.75 \\
& 7.7856(10), 10.1191(12), 14.647(2) \\
& 91.391(10), 101.045(9), 110.180(8) \\
& 1057.9(2) \\
& 1 \\
& 1.76 \\
& 0.16 \times 0.14 \times 0.04
\end{aligned}
$$

$0.693,0.746$

$19008,4740,3826$

0.040

0.650

$0.038,0.079,1.02$

4740

271

0

$-1.33,1.89$

$\mathrm{H}$ atoms were placed using standard geometric models with their displacement parameters riding on those of their parent atoms. The crystal structure of ligand (1) is partially disordered. Specifically the terminal part of the alkyl chain and the disulfide five-membered ring were split in two positions and refined with constraints and restraints (SAME, EXYZ and EADP). Molecular graphics were obtained with Mercury3.8 (Macrae et al., 2006). Crystal data, data collection and structure refinement details for ligand (1) and cocrystal (1).(2) are summarized in Table 1.

2.5. Synthesis of gold nanoparticles AuNP-(1)

Gold nanoparticles (AuNPs) stabilized with dodecanethiol (DT), AuNP-DT, were synthesized using a slightly modified Brust method (Dass et al., 2008). $\mathrm{HAuCl}_{4}$ dissolved in milli-Q Water (mQW) was added to a solution of tetraoctylammonium bromide in toluene and stirred at room

$[M+\mathrm{Na}]^{+}$502.9. Elemental analysis: calc. for $\mathrm{C}_{14} \mathrm{H}_{13} \mathrm{~F}_{4} \mathrm{I}_{1} \mathrm{O}_{2} \mathrm{~S}_{2}$ : C 35.01, H 2.73, F 15.82, S 13.35; found: C 35.16, H 2.8, F 15.73 , S $15.5 \%$.

\subsection{General procedure for the synthesis of the cocrystal}

(1) $\cdot(2)$

The formation of the cocrystal (1).(2) was performed by mixing the XB donor ligand (1) with XB acceptor moiety (2) in chloroform in a 2:1 molar ratio. After $3 \mathrm{~d}$ at room temperature, pale yellow block crystals were formed. Melting point: 385-387 K FT-IR pure 1,2-di(4-pyridyl)ethylene (2) ( $\mathrm{cm}^{-1}$, selected bands): 3025.9, 1594, 1410.4, 991, 820. Cocrystal (1).(2): IR ( $\mathrm{cm}^{-1}$, selected bands): 3029, 2935.6, 1789.4, 1603.3, 1479.8, 1087.2, 979.6, 830.8, 796.

\subsection{Structural analysis}

Single-crystal X-ray data were collected on a Bruker KAPPA APEX II diffractometer with Mo $K \alpha$ radiation $(\lambda=$ 0.71073 ) and CCD detector. Data were collected at low temperature $(100 \mathrm{~K})$ using a Bruker KRYOFLEX device. Data collection and reduction were performed by SMART and SAINT (Bruker, 2012) and absorption correction, based on multi-scan procedure, by SADABS (Bruker, 2012). The structures were solved by SHELXS (Sheldrick, 2008) and refined by SHELXL (Sheldrick, 2015), respectively. The refinement was carried out by full-matrix least-squares on $F^{2}$. temperature until the gold precursors were transferred into the organic phase. The nucleation of gold clusters in toluene was mediated by the addition of sodium borohydride in $\mathrm{mQW}$ as a reducing agent, under vigorous stirring for $1 \mathrm{~h}$. The aqueous phase was removed and the collected organic phase was stirred at room temperature overnight. The bromide ions were displaced by the addition of DT, and the mixture was incubated at $338 \mathrm{~K}$ for $2 \mathrm{~h}$. After cooling, the reaction mixture was purified with several cycles of centrifugation at 9500 r.p.m. The synthesized AuNP-DT were characterized by TEM, DLS, UV-vis and FT-IR before use. In the ligand-exchange reaction ligand (1) was added to a dispersion of AuNP-DT in toluene under moderate stirring. The mixture was then left at room temperature for $18 \mathrm{~h}$ under vigorous stirring, followed by purification with several cycles of centrifugation at 9500 r.p.m. The resulting AuNP-DT/XB were characterized by TEM, DLS, UV-vis and FT-IR investigation before use. AuNP concentration was obtained by calculations based on $\mathrm{Au}$ content (obtained by ICP-AES measurements) and NP size (obtained by TEM). ICP-AES was also used to evaluate the amount of iodine on the gold surface after the ligandexchange reaction.

\section{Results and discussion}

Ligand (1) bears two important chemical functionalities: an effective XB donor group, the 4-iodotetrafluorobenzene, and 


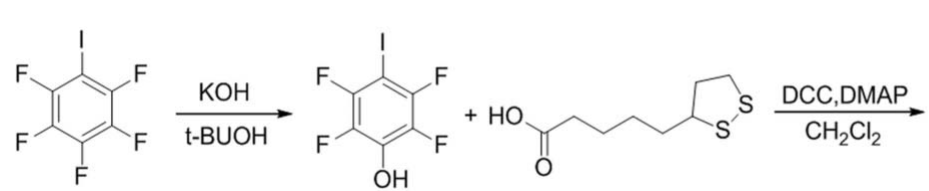

Figure 2

Synthetic scheme for the formation of ligand (1).

an efficient binding moiety to gold surfaces, the thioctic group (see Fig. 2). The 4-iodotetrafluorobenzene is a well known XB donor and thanks to its efficiency in forming very short and directional XBs it has been widely used to promote the construction of a great variety of halogen-bonding complexes (Baldrighi et al., 2010; Aakeröy et al., 2007; Takemura et al., 2014). In addition, 4-iodotetrafluorobenzene can be easily functionalized with different organic pendants without using complex synthetic procedures (Dumele et al., 2015; Bruce et al., 2010). Thus, it is clear that 4-iodotetrafluorobenzene is a versatile building block both in covalent and supramolecular chemistry and for this reason it was selected as an $\mathrm{XB}$ donor unit in ligand (1). The thioctic acid was instead chosen as a linking molecule that simultaneously allows binding to the $\mathrm{XB}$ donor unit and to a gold surface through the acid and the disulfide groups, respectively.

Specifically, ligand (1) was obtained in a good yield first substituting one $\mathrm{F}$ atom on the aromatic ring of the 4-iodotetrafluorobenzene with a hydroxyl group, and then the formed alcohol was used for an esterification reaction of the thioctic acid to form ligand (1) (Fig. 2).

Before studying the ability of (1) to interact with $\mathrm{XB}$ acceptors we characterized the solid-state assembly of ligand (1) (Table 1). Ligand (1) is indeed a self-complementary system with both $\mathrm{XB}$ donor and acceptor groups present in its structure. In fact, it is expected that the I atom works as an XB donor, while the $\mathrm{O}$ and/or $\mathrm{S}$ atoms act as electron-density donor units. Solid-state analysis showed that ligand (1) selfassembled in an infinite halogen-bonded chain driven by contacts occurring between the I atoms and one of the $\mathrm{S}$ atoms [S1 $(2+x, 1+y, 1+z)]$ on the disulfide bridge (Fig. 3 top). The geometrical parameters of the interaction are the following: S...I distance 3.2305 (10) $\AA$ and C-I . . S angle $175.65(9)^{\circ}$.

Although it is known that neutral S atoms may act as good XB acceptors, structures assembled through an S...I synthon, in which the $\mathrm{S}$ atom is in a disulfide bridge, are rare and only a few examples are reported in the Cambridge Structural Database [CSD codes: XESTIZ (Takemura et al., 2014), IFOSUL (Bjorvatten et al., 1962), IFOSUL01 (Wolstenholme et al., 2006) and NEPJIB (Makarov et al., 2001)]. In addition, in these crystal structures the XBs are weak, namely long, with $\mathrm{C}-\mathrm{I} \cdots \mathrm{S}$ angles quite far away from linearity. Unlike to the reported systems, the structure of ligand (1) reveals that when a strong and efficient $\mathrm{XB}$ donor is used the $\mathrm{S}$ atom in the disulfide bridge acts as a good $\mathrm{XB}$ acceptor and the $\mathrm{S}-\mathrm{S} \cdots \mathrm{I}$ synthon becomes the structure-determining factor of the

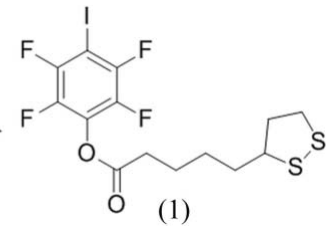

system. Specifically, the XB distance in (1) is much shorter than in the examples reported in the CSD and corresponds to a $15 \%$ reduction of the sum of van der Waals $(\mathrm{vdW})$ radii of the $\mathrm{XB}$ donor and acceptor. Another interesting aspect in this structure is the selectivity of the $\mathrm{XB}$ donor towards a specific electron-density donor site. In fact, although there are two $\mathrm{O}$ atoms, which in principle are able to interact with the $\sigma$-hole on the I atom, the XB exclusively occurs with the lone pair of the $\mathrm{S}$ atom in the disulfide bridge. This selectivity has been investigated by varying both the crystallization solvent and the crystallization temperature. Notably, in all the experiments, the S . . I synthon was preferred over the $\mathrm{O} \cdots \mathrm{I}$ synthon. A possible explanation of this specificity could be found in the hard and soft acids and bases theory (HSAB), applied to the halogen-bonded complexes, where soft acids preferentially interact with soft bases and vice versa (Laurence \& Gal, 2009). In (1) the I atom is a soft acid, in fact it is the most polarizable, or least electronegative, atom of the halogen series and thus possesses a very soft electron cloud. On the other hand, between the halogen-bonding acceptors the less electronegative site, thus the softer base, is the $\mathrm{S}$ atom. Thus, it is expected that the lone pairs of the $\mathrm{S}$ atom, further from the nuclei than those of the oxygen, more easily interact with the positive hole of the I atom as observed in (1). This observation nicely mirrors what Laurence and Gal reported on the structure-basicity relationship of the $\mathrm{XB}$ acceptors. In fact, for a halogen-bonded complex involving $\mathrm{I}_{2}$ and 1,2-dithiane a negative $\Delta G^{\circ}$ value

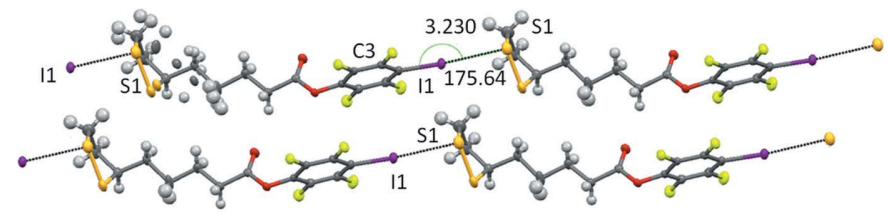

(a)

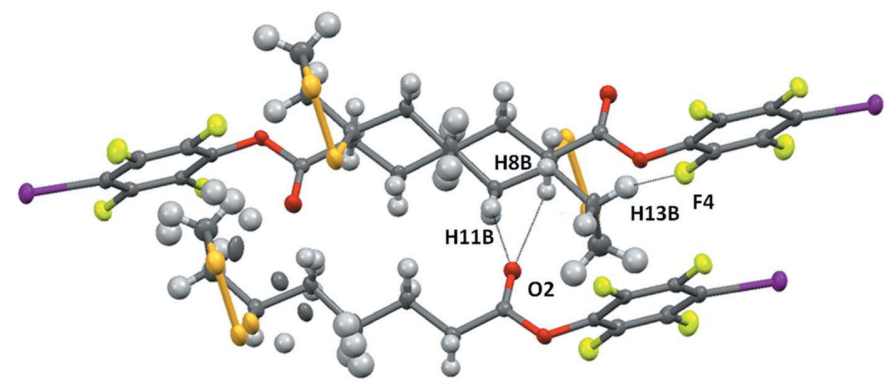

(b)

Figure 3

(a) Representation of the infinite halogen-bonded chain (displacement ellipsoids are drawn at $50 \%$ probability level) in the self-complementary XB donor ligand (1). (b) Crystal packing of ligand (1) where weak hydrogen-bonding contacts are highlighted. Colour code: grey, carbon; green, fluorine; red, oxygen; white, hydrogen; magenta, iodine; yellow, sulfur. XBs and HBs are shown as black dotted lines. The distance and angle for the halogen bonding are given. The disorder of the disulfide ring and of the terminal part for the alkyl chain is shown. 
was experimentally found (Nelander et al., 1972), while a positive one was reported when the electron density donor site was an ester moiety (Laurence et al., 1979). These findings suggest that the $\mathrm{S}-\mathrm{S}$ group is a better XB acceptor than the ester moiety and might explain the observed selectivity towards the formation of the S...I synthon over the O $\cdots$ I synthon in (1).

The overall crystal packing of (1) is also stabilized by other short contacts. The $\mathrm{O}$ atom of the carbonyl unit behaves as a bifurcated hydrogen-bonding acceptor towards two $\mathrm{H}$ atoms of different molecules [distances: $\mathrm{O} 2 \cdots \mathrm{H} 8 B(1+x, y, z)$ $2.473 \AA$ and $\mathrm{O} 2 \cdots \mathrm{H} 11 B(-x, 1-y, z) 2.524 \AA$ ] , while the $\mathrm{F}$ atoms on the aromatic ring weakly interact with several $\mathrm{H}$ atoms belonging either to the five-membered ring or the alkyl chain of adjacent molecules (Fig. $3 b$ ). The $\mathrm{F}$ atoms are also involved in weak F...F contacts.

The supramolecular assembly capability of ligand (1), namely the ability to form XBs, was tested using 1,2-di(4pyridyl)ethylene (2) as an $\mathrm{XB}$ acceptor. The choice of this $\mathrm{XB}$ acceptor was driven by two main factors: (i) to use a simple structural system to minimize steric requirements and other competitive non-covalent interactions, and (ii) to have an electron-density donor group that could mimic a solvent molecule with $\mathrm{XB}$ acceptor ability such as pyridine.

As mentioned previously, ligand (1) is a self-complementary system, therefore the construction of the supramolecular adduct with the $\mathrm{XB}$ acceptor (2) has to get through a competitive process that involves the rupture of the $\mathrm{S} \cdots \mathrm{I}$ synthon followed by the formation of the N...I XB contact. Pyridyl $\mathrm{N}$ atoms are very good Lewis bases, better than $\mathrm{S}-\mathrm{S}$ groups (Laurence \& Gal, 2009), and the N...I synthon has been widely used in crystal engineering driven by XB and, in particular, the 4-iodotetrafluorobenzene and pyridyl derivatives have been reported to give halogen-bonded cocrystals with structure dominated by short and directional $\mathrm{N} \cdot$. I contacts (Aakeröy et al., 2015; Cariati et al., 2011). Based on these considerations we expect that the $\mathrm{N}$...I synthon could replace S...I interactions and promote the formation of halogen-bonded adducts.

The formation of the supramolecular system was obtained by mixing the $\mathrm{XB}$ acceptor and donor in a $1: 2$ ratio in $\mathrm{CHCl}_{3}$ solution and, molecule (2) being a ditopic XB acceptor, it was expected that a trimeric halogen-bonded adduct would be obtained. A preliminary indication of the formation of a new two-component supramolecular system was provided by the melting point of the crystalline powder obtained from the crystallization, which was different from the melting points of the pure starting molecules (1) and (2) [385-387 K for the complex (1).(2), 359-361 K for (1) and 421-423 K for (2)]. Further characterization of the complex was performed using IR spectroscopy. IR has been proven to be a valuable and fast technique to detect the occurrence of $\mathrm{XB}$, indeed it is well known that the formation of intermolecular interactions between donor and acceptor species affects the vibrational motions in terms of intensity and frequency shift. Specifically, in the adduct (1).(2) the $v_{\mathrm{C}-\mathrm{H}}$ stretching of the pyridine ring became less intense and was shifted compared with the pure
$\mathrm{XB}$ acceptor, $3039 \mathrm{~cm}^{-1}$ in (1).(2) and $3026 \mathrm{~cm}^{-1}$ in (2), respectively (Fig. S1). This behavior is the result of a reduction in electron density on the pyridyl ring that occurs during the formation of the halogen-bonding contact. Even the vibration bands related to the 4-iodotetrafluorobenzene underwent small variations in the complex (1) $(2)$, specifically the $\mathrm{C}-\mathrm{F}$ stretching band is red-shifted compared with the signals of the pure ligand (1), $1087 \mathrm{~cm}^{-1}$ in the XB adduct and $1112 \mathrm{~cm}^{-1}$ in (1). This phenomenon is associated with an increase in electron density on the fluorinated aromatic ring of the module (1) upon formation of the XB contact. All these changes in the IR spectrum of the complex (1).(2) are similar to those observed in several halogen-bonded complexes obtained using 4-iodotetrafluorobenzene and pyridine (Cavallo et al., 2016) as building units. This finding suggests that the analysed system is not a simple mechanical mixture of the two components, but it is a new supramolecular entity assembled thanks to the halogen bond.

To further verify the presence of the halogen-bonded complex and carefully analyze the assembly capability of (1), we performed an X-ray diffraction study on the (1).(2) cocrystal. Single crystals suitable for X-ray diffraction analysis were grown by slow isothermal evaporation from a $\mathrm{CHCl}_{3}$ solution containing a 2:1 mixture of the $\mathrm{XB}$ donor ligand (1) and acceptor (2) in $3 \mathrm{~d}$ at room temperature (Table 1). As expected, the X-ray analysis confirmed the formation of the trimeric halogen-bonded complex (1).(2) and highlighted that the assembling of the two molecules was mainly driven by the N...I synthon (Fig. 4a). Moreover, the observed XB is quite short and linear with geometrical parameters similar to those reported for other halogen-bonded systems involving 4-iodotetrafluorobenzene-pyridine derivatives (Aakeröy et al., 2015; Cariati et al., 2011): N $\cdots I=2.749$ (3) $\AA$, $22 \%$ reduction on the sum of vdWs radii, and $\mathrm{C}-\mathrm{I} \cdots \mathrm{N}$ angle $177.48(12)^{\circ}$. These features highlight that the N...I synthon can easily overcome the I...S synthon, and the two modules, 4-iodotetra-

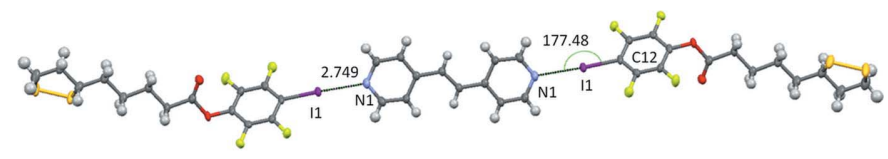

(a)

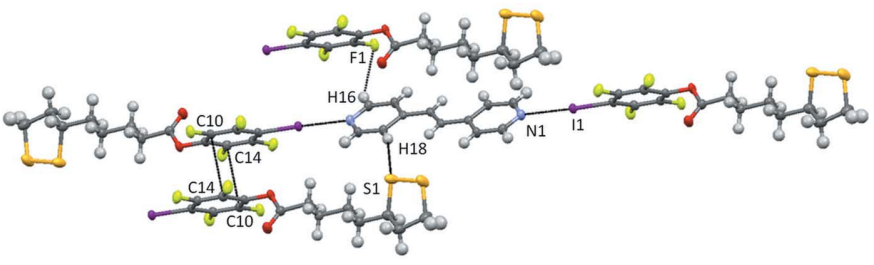

(b)

Figure 4

(a) Single-crystal X-ray trimeric structure of complex (1).(2) (displacement ellipsoids are drawn at $50 \%$ probability). (b) Partial representation of the crystal packing of (1).(2), where $\pi \cdots \pi$, S $\cdots H$ and $H \cdots F$ weak contacts are shown. Colour code: grey, carbon; green, fluorine; red, oxygen; white, hydrogen; magenta, iodine; yellow, sulfur; blue, nitrogen. XBs and other interactions are shown as black dotted lines. The distance and angle for the halogen bonding are given. 


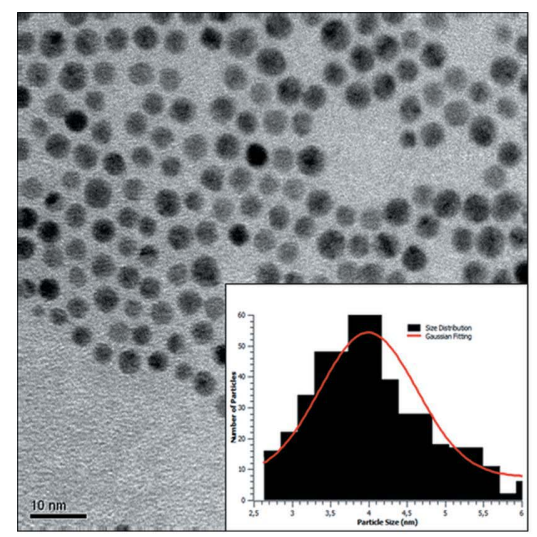

(a)

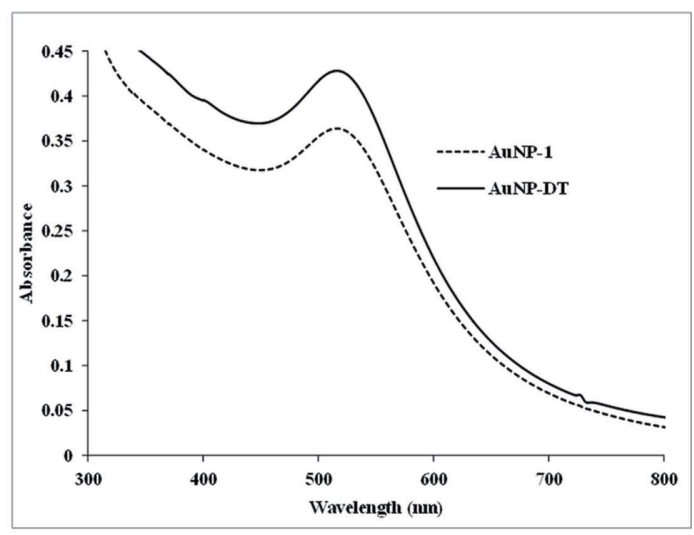

(b)

Figure 5

(a) A representative TEM image of AuNP-(1) showing NP size distribution (inset) resulting in NP averaged diameter of $4 \mathrm{~nm}$. (b) UV-vis spectra showing the surface plasmon absorption bands of AuNP(1) and AuNP-DT dispersions.

fluorobenzene-pyridine, are efficient building units in promoting the formation of halogen-bonded adducts.

In addition to XB, crystal packing of the cocrystal (1) (2) was promoted by the $\pi \cdots \pi$ interactions occurring between two adjacent tetrafluoroaromatic rings (ring centroid distance of $3.488 \AA$ ) and by several S. .H and H...F weak contacts that further stabilize the entire system (Fig. $4 b$ ).

Having established the ability of ligand (1) to behave as an efficient XB donor, we attempted the synthesis of gold NPs functionalized with (1). The formation of the AuNP-(1) was performed starting from AuNPs capped with dodecanthiol (AuNP-DT), which were subjected to a ligand exchange reaction in which the alkyl thiol moieties were partially substituted with ligand (1) (see $\$ 2$ for details, Fig. S2). The presence of the iodinated ligand on the NP surface was confirmed by ICP-AES analysis (detection of Au and I), which showed approximately $70 \%$ exchange with the XB donor (1). Notably, TEM images showed high spherical size homogeneity of the AuNP-(1) with an average diameter of $4 \mathrm{~nm}$ (Fig. 5a). Further characterizations were performed using UV-vis measurements: both AuNP-DT and AuNP-(1) showed a surface plasmon peak at $\lambda_{\max } \simeq 513 \mathrm{~nm}$ in toluene; such
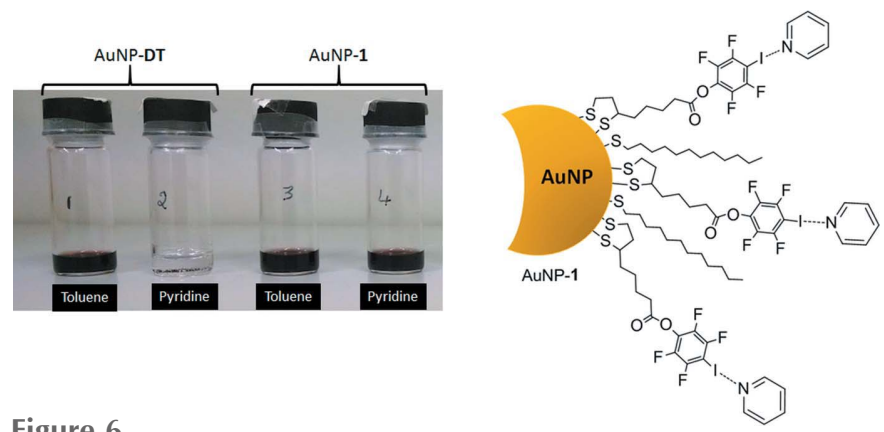

Figure 6

(b) Vials showing the different dispersibility profile in toluene and pyridine for AuNP-DT and AuNP-(1). (b) Schematic representation of the halogen-bonding interactions occurring between the $\mathrm{XB}$ donor groups on the AuNP surface and the solvent pyridine. optical behaviour is in line with TEM results and in agreement with the literature for similar NPs (Fig. $5 b$; Shirman et al., 2010). DLS investigation confirmed these results showing that beyond a population of $\sim 4 \mathrm{~nm}$ hydrodynamic size, a population at about $60 \mathrm{~nm}$ was also present, probably related to NP agglomerates. This second population was present in both samples, but it was more accentuated in AuNP-(1) dispersions (Fig. S3). Overall, these results highlighted that substitution of DT with ligand (1) did not strongly affect the structure of the NPs and their dispersion in toluene.

Finally, AuNP-(1) was tested in a solvent having XB acceptor capability such as pyridine to evaluate the ability of the $\mathrm{XB}$ donor surface ligand to improve their dispersibility. As observed in the UV-vis experiments, both AuNP-DT and AuNP-(1) were well dispersed in toluene, forming stable dispersions. The two NPs showed a different behaviour when dispersed in pyridine. Specifically, the NPs functionalized with DT chains were insoluble in pyridine, while AuNP-(1) gave a stable dispersion (Fig. 6). However, DLS showed that the AuNP-(1) dispersion in pyridine was dominated by a single population of larger NP agglomerates of $\sim 100 \mathrm{~nm}$ hydrodynamic size (Fig. S3). This behaviour can be explained in terms of halogen-bonding interactions between the solvent molecules and the NPs. It is reasonable to think that the pyridine molecules actively interact through XBs with the I atoms on the gold surface of AuNP-(1) facilitating the dispersion of the modified NPs in pyridine (Fig. 6). This hypothesis is supported by the structural results on the $\mathrm{XB}$ donor capability of (1), where the XB interaction between the I atom and the pyridyl derivative was observed and identified as a strong XB interaction. Further support to this explanation can be found in other research on $\mathrm{XB}$, which show that the N. . I synthon formed by two building units used is present in solution (Carlsson et al., 2015; Erdélyi, 2012). This evidence supports the fact that the $\mathrm{XB}$ formed on the ligand layer can substantially influence the dispersibility profile of AuNP-(1). Differently the lack of XB donor groups on AuNP-DT does not allow the formation of $\mathrm{XB}$ interactions resulting in a highly aggregated system, which precipitates in pyridine.

\section{Conclusions}

We have reported the synthesis of a new halogen-bonding donor ligand (1) that can be easily used for the functionalization of gold NP surfaces. Ligand (1) turned out to be a very efficient building block in covalent synthesis and in supramolecular chemistry. While the X-ray analysis on ligand (1) and its cocrystal (1).(2) has, once again, highlighted the ability 
of the 4-iodotetrafluorobenzene moiety to behave as a powerful $\mathrm{XB}$ donor, the disulfide bridge has successfully allowed the anchoring of (1) on the gold surface leading to the formation of a new multicomponent nanosystem with $\mathrm{XB}$ donor functionalities, AuNP-(1). The preliminary experiments on dispersibility of AuNP-(1) have shown a possible active role of the halogen bond in facilitating the dispersion of modified NPs in pyridine if compared with NPs functionalized with the dodecanethiol. We believe that the XB donor ligand (1) is a very versatile unit to functionalize gold nanoparticles and that the 4-iodotetrafluorobenzene moiety can drive the dispersion and/or assembling of gold nanoparticles under halogen-bonding control.

\section{References}

Aakeröy, C. B., Schultheiss, N., Desper, J. \& Moore, C. (2007). CrystEngComm, 9, 421-426.

Aakeröy, C. B., Spartz, C. L., Dembowski, S., Dwyre, S. \& Desper, J. (2015). IUCrJ, 2, 498-510.

Astruc, D., Lu, F. \& Aranzaes, J. R. (2005). Angew. Chem. Int. Ed. 44, 7852-7872.

Baldrighi, M., Metrangolo, P., Meyer, F., Pilati, T., Proserpio, D., Resnati, G. \& Terraneo, G. (2010). J. Fluor. Chem. 131, 1218-1224.

Bjorvatten, T., Bäckström, R., Mörk, P. C., Sim, G. A., Theander, O. \& Flood, H. (1962). Acta Chem. Scand. 16, 749-754.

Bruce, D. W., Metrangolo, P., Meyer, F., Pilati, T., Präsang, C., Resnati, G., Terraneo, G., Wainwright, S. G. \& Whitwood, A. C. (2010). Chem. Eur. J. 16, 9511-9524.

Bruker (2012). APEX2, SADABS, SAINT. Bruker AXS Inc., Madison, Wisconsin, USA.

Burda, C., Chen, X., Narayanan, R. \& El-Sayed, M. A. (2005). Chem. Rev. 105, 1025-1102.

Cariati, E., Cavallo, G., Forni, A., Leem, G., Metrangolo, P., Meyer, F., Pilati, T., Resnati, G., Righetto, S., Terraneo, G. \& Tordin, E. (2011). Cryst. Growth Des. 11, 5642-5648.

Carlsson, A.-C. C., Veiga, A. X. \& Erdélyi, M. (2015). Halogen Bonding II: Impact on Materials Chemistry and Life Sciences, edited by P. Metrangolo \& G. Resnati, pp. 49-76. Cham: Springer International Publishing.

Cavallo, G., Metrangolo, P., Milani, R., Pilati, T., Priimägi, A., Resnati, G. \& Terraneo, G. (2016). Chem. Rev. 116, 2478-2601.

Coulston, R. J., Jones, S. T., Lee, T.-C., Appel, E. A. \& Scherman, O. A. (2011). Chem. Commun. 47, 164-166.

Daniel, M. C. \& Astruc, D. (2004). Chem. Rev. 104, 293-346.

Dass, A., Guo, R., Tracy, J. B., Balasubramanian, R., Douglas, A. D. \& Murray, R. W. (2008). Langmuir, 24, 310-315.

Derjaguin, B. \& Sidorenkov, G. (1941). C. R. Acad. Sci. 32, 622-626.

Dumele, O., Trapp, N. \& Diederich, F. (2015). Angew. Chem. Int. Ed. 54, 12339-12344.

Erdélyi, M. (2012). Chem. Soc. Rev. 41, 3547-3557.

Farrugia, L. J. (1997). J. Appl. Cryst. 30, 565.
Ghosh, P., Han, G., De, M., Kim, C. K. \& Rotello, V. M. (2008). Adv. Drug Deliv. Rev. 60, 1307-1315.

Grzelczak, M., Pérez-Juste, J., Mulvaney, P. \& Liz-Marzán, L. M. (2008). Chem. Soc. Rev. 37, 1783-1791.

Gupta, A. K. \& Gupta, M. (2005). Biomaterials, 26, 3995-4021.

Hao, E. \& Lian, T. (2000). Chem. Mater. 12, 3392-3396.

Heo, K., Miesch, C., Emrick, T. \& Hayward, R. C. (2013). Nano Lett. 13, 5297-5302.

Khanna, P. K., Gaikwad, S., Adhyapak, P. V., Singh, N. \& Marimuthu, R. (2007). Mater. Lett. 61, 4711-4714.

Laurence, C. \& Gal, J. F. (2009). Lewis Basicity and Affinity Scales: Data and Measurement. New York: Wiley.

Laurence, C., Guiheneuf, G. \& Wojtkowiak, B. (1979). J. Am. Chem. Soc. 101, 4793-4801.

Li, H., Chen, D.-X., Sun, Y.-L., Zheng, Y. B., Tan, L.-L., Weiss, P. S. \& Yang, Y.-W. (2013). J. Am. Chem. Soc. 135, 1570-1576.

Macrae, C. F., Edgington, P. R., McCabe, P., Pidcock, E., Shields, G. P., Taylor, R., Towler, M. \& van de Streek, J. (2006). J. Appl. Cryst. 39, 453-457.

Makarov, A. Y., Shakirov, M. M., Bagryanskaya, I. Y., Gatilov, Y. V., Zibarev, A. V. \& Shuvaev, K. V. (2001). Chem. Commun. pp. 17741775.

Nelander, B., Norén, I., Rømming, C., Andresen, A. F., Pearson, W. B. \& Meisalo, V. (1972). Acta Chem. Scand. 26, 809-814.

Pankhurst, Q. A., Connolly, J., Jones, S. K. \& Dobson, J. (2003). J. Phys. D, 36, R167-R181.

Rycenga, M., Cobley, C. M., Zeng, J., Li, W., Moran, C. H., Zhang, Q., Qin, D. \& Xia, Y. (2011). Chem. Rev. 111, 3669-3712.

Saha, K., Agasti, S., Kim, C., Li, X. \& Rotello, V. (2012). Chem. Rev. 112, 2739-2779.

Sheldrick, G. M. (2008). Acta Cryst. A64, 112-122.

Sheldrick, G. M. (2015). Acta Cryst. C71, 3-8.

Shirman, T., Arad, T. \& van der Boom, M. E. (2010). Angew. Chem. Int. Ed. 49, 926-929.

Shirman, T., Kaminker, R., Freeman, D. \& van der Boom, M. E. (2011). ACS Nano, 5, 6553-6563.

Sperling, R. \& Parak, W. J. (2010). Philos. Trans. A. Math. Phys. Eng. Sci. 368, 1333-1383.

Takemura, A., McAllister, L. J., Karadakov, P. B., Pridmore, N. E., Whitwood, A. C. \& Bruce, D. W. (2014). CrystEngComm, 16, 42544264.

Verwe, E. J. W. \& Overbeek, J. T. G. (1948). Theory of the Stability of Lyophobic Colloids. Amsterdam: Elsevier.

Vuluga, D., Legros, J., Crousse, B. \& Bonnet-Delpon, D. (2008). Chem. Commun. pp. 4954-4955.

Wolstenholme, D. J., Robertson, K. N., Gonzalez, E. M. \& Cameron, T. S. (2006). J. Phys. Chem. A, 110, 12636-12643.

Wu, L., Li, Q., Wu, C. H., Zhu, H., Mendoza-Garcia, A., Shen, B., Guo, J. \& Sun, S. (2015). J. Am. Chem. Soc. 137, 70717074.

Xu, L., Ma, W., Wang, L., Xu, C., Kuang, H. \& Kotov, N. A. (2013). Chem. Soc. Rev. 42, 3114-3126.

Yang, Y., Sun, Y. \& Song, N. (2014). Acc. Chem. Res. 47, 1950-1960.

Zhang, J., Santos, P. J., Gabrys, P. A., Lee, S., Liu, C. \& Macfarlane, R. J. (2016). J. Am. Chem. Soc. 138, 16228-16231. 